\title{
Computational design of model Re/Ru bearing Ni-base superalloys
}

\author{
K.V. Vamsi ${ }^{1, a}$, K.N. Goswami ${ }^{1,2}$, K.S. Vinay ${ }^{1}$, S.K. Verma ${ }^{1}$, R. Balamuralikrishnan ${ }^{3}$, Niranjan Das ${ }^{3}$, D. Banerjee ${ }^{1}$, \\ and S. Karthikeyan ${ }^{1}$ \\ ${ }^{1}$ Department of Materials Engineering, Indian Institute of Science, Bangalore 560012, India \\ ${ }^{2}$ School of Metallurgy and Materials, University of Birmingham, Edgbaston, UK \\ ${ }^{3}$ Defence Metallurgical Research Laboratory, Kanchanbagh, Hyderabad 500058, India
}

\begin{abstract}
It is well established that Re and $\mathrm{Ru}$ additions to Ni-base superalloys result in improved creep performance and phase stability. However, the role of $\mathrm{Re}$ and $\mathrm{Ru}$ and their synergetic effects are not well understood, and the first step in understanding these effects is to design alloys with controlled microstructural parameters. A computational approach was undertaken in the present work for designing model alloys with varying levels of Re and $\mathrm{Ru}$. Thermodynamic and first principles calculations were employed complimentarily to design a set of alloys with varying Re and Ru levels, but which were constrained by constant microstructural parameters, i.e., phase fractions and lattice misfit across the alloys. Three ternary/quaternary alloys of type Ni-Al$x R e-y R u$ were thus designed. These compositions were subsequently cast, homogenized and aged. Experimental results suggest that while the measured volume fraction matches the predicted value in the Ru containing alloy, volume fraction is significantly higher than the designed value in the Re containing alloys. This is possibly due to errors in the thermodynamic database used to predict phase fraction and composition. These errors are also reflected in the mismatch between predicted and measured values of misfit.
\end{abstract}

\section{Introduction}

Modifications to blade design and evolution of alloy composition over the past 60 years have enabled turbine components made of Ni-base superalloys to operate at higher temperatures enabling greater thrust and engine efficiency [1]. The design transition from wrought to directionally solidified and single crystal blades, the introduction of intricate forced-air cooling schemes, and development of environment and thermal barrier coatings are partly responsible for enhancement in operating temperatures. Changes to alloy composition have also positively impacted the high temperature performance. Alloying additions affect mechanical properties either directly (such as via solute strengthening, changing diffusivities, stacking fault energies and elastic moduli) or indirectly via alterations to microstructural parameters (volume fraction, misfit and size of the precipitates). Addition of slow diffusing elements like W, Mo and Re (beginning with II $^{\text {nd }}$ generation alloys) has led to remarkable improvements in creep resistance over first generation alloys which are Re-free. The recent $\mathrm{V}^{\text {th }}$ generation alloys additionally contain high amounts of $\mathrm{Ru}(\sim 6 \mathrm{wt} \%)$ and this has resulted in dramatic life enhancement. While simultaneous presence of Re and Ru in $\mathrm{IV}^{\text {th }}$ and $\mathrm{V}^{\text {th }}$ generation alloys results in significant benefits, both these metals are expensive and their availability in earth's crust is limited [2]. This has driven research towards developing $\mathrm{Ni}$-base alloys which are

\footnotetext{
${ }^{a}$ Corresponding author: karthik@materials.iisc.ernet.in
}

either $\mathrm{Re} / \mathrm{Ru}$ free or leaner in these elements, but which additionally do not compromise mechanical response and phase stability at high temperatures significantly. In this context, recent research has focused on understanding individual and synergistic effects of $\mathrm{Re}$ and $\mathrm{Ru}$ so that these may be replicated with other alloying elements. These studies typically involve systematic variation of $\mathrm{Ru}$ and $\mathrm{Re}$ amounts in model alloys, and characterization of their effect on microstructure and properties [3-12].

It is understood that while $\mathrm{Re}$ improves creep resistance via solute strengthening and due to lower diffusivity [13], Ru suppressed the formation of Topologically Close Packed (TCP) phases which would otherwise form in Re containing alloys [8-10]. This latter effect is not well understood. Previous studies by Ofori et al. and Pyczak et al. $[11,12]$ show that Ru supresses TCP formation by causing a "reverse partitioning" of Re to $\gamma^{\prime}$ which in the absence of $\mathrm{Ru}$ partitions strongly to $\gamma$. In contrast, Volek et al. [3] present evidence that shows that such reverse partitioning does not take place.

A possible reason for such contrasting conclusions from these studies is that these alloys were designed such that while systematically varying the relative amounts of $\mathrm{Re}$ and $\mathrm{Ru}$, no constraints were imposed on microstructure. For example, such a variation in chemistry across the model alloys could result in a variation in phase fractions, precipitate size and lattice misfit between $\gamma$ and $\gamma^{\prime}$ across the alloys. With this microstructural variation across the alloys, it is incorrect to assign the observed mechanical response purely to a change in composition. In other

This is an Open Access article distributed under the terms of the Creative Commons Attribution License 4.0, which permits unrestricted use, distribution, and reproduction in any medium, provided the original work is properly cited. 
words, it is unclear whether the effect of $\mathrm{Re}$ and $\mathrm{Ru}$ are primarily microstructural or if there is a direct effect unique to these alloying elements. If the effect is the former, then it may be possible to replace $\mathrm{Re}$ and $\mathrm{Ru}$ with elements which produce similar changes to the microstructure. However, if the effects of Re and $\mathrm{Ru}$ are direct, such as their role in phase stability, diffusivity and stacking fault energy, then other paradigms for replacing $\mathrm{Re}$ and Ru need to be considered.

The present study is aimed at designing alloy chemistries which would enable the decoupling of direct and microstructural effects of $\mathrm{Re}$ and $\mathrm{Ru}$. The aim of this work was to explore if one can computationally design a series of alloys with varying levels of $\mathrm{Re}$ and $\mathrm{Ru}$, but where microstructural parameters such as $\gamma^{\prime}$ phase fractions and lattice misfit are constant across the alloys. The computational design approach involved thermodynamic and electronic structure calculations using Thermo-Calc $^{\mathrm{TM}}$ [14] and Vienna Ab-initio Simulation Package (VASP) [15-18], respectively. These techniques were used complementarily to design a set of model alloys with varying $\mathrm{Re}$ and $\mathrm{Ru}$ levels, but which are constrained by $\gamma^{\prime}$ phase fractions and lattice misfit. Three alloys of type Ni-Al-xRe-yRu were designed. Subsequently, experiments were conducted to test if the design methodology was valid or not.

The details of the design approach are elaborated on in the next section. The designed alloy compositions were cast, homogenized and aged and these details are presented subsequently. In the final section of this paper, the experimental validation of alloy design is presented and discussed.

\section{Alloy design methodology}

There is extensive literature on creep of Ni-base blade alloys and these suggest that precipitate $\left(\gamma^{\prime}\right)$ volume fraction and misfit between the $\gamma^{\prime}$ and $\gamma$ phases are important microstructural parameters which govern creep life in Ni-base superalloys $[19,20]$. It has been shown that a $\gamma^{\prime}$ volume fraction of $\sim 60 \%$ and large negative misfit $\left(\sim-0.003\right.$ in $\mathrm{IV}^{\text {th }}$ generation alloys $)$ are beneficial. Given the importance of these parameters on creep, three model (ternary/quaternary) alloys with varying $\mathrm{Re}$ and $\mathrm{Ru}$ levels were designed such that, across the alloys:

1. misfit at $1050{ }^{\circ} \mathrm{C}$ was constant (i.e., typical creep temperature),

2. $\gamma^{\prime}$ volume fraction at $1050{ }^{\circ} \mathrm{C}$ was constant, and

3. the amount of Re and Ru was less than $6 \mathrm{wt} \%$ each.

Thermo-Calc ${ }^{\mathrm{TM}}$ software [14] in conjunction with the TTNI8 database for Ni-base superalloys was used to compute the volume fraction of $\gamma^{\prime}$ and the composition of $\gamma$ and $\gamma^{\prime}$ at $1050{ }^{\circ} \mathrm{C}$. With $\mathrm{Ni}-10 \mathrm{Al}$ (amounts in wt\%) as the base composition, several alloys ( $\sim 25$ compositions) were explored with varying $\mathrm{Re}$ and $\mathrm{Ru}$ contents. The variation of $\gamma^{\prime}$ volume fraction with $\mathrm{Re}$ and $\mathrm{Ru}$ levels was mapped out at $1050{ }^{\circ} \mathrm{C}$ and this is indicated in the contour plot shown in Fig. 1a. The compositions of $\gamma$ and $\gamma^{\prime}$ at $1050{ }^{\circ} \mathrm{C}$ were also determined for the 25 compositions.
For calculating misfit, Vegard's coefficients of various alloying elements in $\gamma$ and $\gamma^{\prime}$ were computed from first principles calculations. The Vienna Ab-initio Simulation Package (VASP 4.6) [15-18] working within the MedeA ${ }^{\circledR}$ platform [21] was used to perform these calculations. The plane wave kinetic energy cut-off of $400 \mathrm{eV}$ and K-point spacing of $0.19-0.20 \AA^{-1}$ was found to be optimal. Projected augmented wave scheme was used to represent the wave functions. Methfessel-Paxton first order spreading was used for Brillouin zone integration [22] and a smearing width of $0.127 \mathrm{eV}$ was used. Pseudopotentials employed were based on Generalized Gradient Approximation (GGA-PBE) and of Perdew-Burke-Ernzerhof [23,24] type. Atomic relaxation was performed using a conjugate gradient method algorithm and calculations were terminated when the Hellmann-Feynman forces were less than $0.02 \mathrm{eV} / \AA$. The parameters used above have been previously shown to be accurate in predicting bulk properties of $\mathrm{Ni}, \mathrm{Al}, \mathrm{Ti}, \mathrm{Ta}$, $\mathrm{Ni}_{3} \mathrm{Al}, \mathrm{Ni}_{3} \mathrm{Ta}$ and $\mathrm{Ni}_{3} \mathrm{Ti}$ [25].

Calculations were performed on a 32-atom supercell of $\mathrm{Ni}_{3} \mathrm{Al}$ in $\mathrm{L1}_{2}$ structure and $\mathrm{Ni}$ in fcc structure. In $\gamma$, one (or more) atom of $\mathrm{Ni}$ was replaced by the alloying element. Similarly, in $\gamma^{\prime}$, an atom of either Ni (sublattice I) or $\mathrm{Al}$ (sublattice II) was replaced by the alloying element. Assuming a linear dependence of equilibrium lattice parameter on the amount of alloying element, Vegard's Coefficients were calculated [26]. $\Gamma_{i}^{\gamma}, \Gamma_{i, I}^{\gamma^{\prime}}$ and $\Gamma_{i, I I}^{\gamma^{\prime}}$ are the Vegard's coefficients of alloying element $i$, in $\gamma$ and in the $\mathrm{Ni}$ and $\mathrm{Al}$ sublattices of $\gamma^{\prime}$ respectively. $\Gamma_{i}^{\gamma}$ was calculated from the slopes of $a_{\gamma}$, the lattice parameter of $\gamma$, as a function of mole fraction, $x_{i}^{\gamma}$ of element $i$ in $\gamma$. Similarly $\Gamma_{i, I}^{\gamma^{\prime}}$ and $\Gamma_{i, I I}^{\gamma^{\prime}}$ were calculated from the slopes of $a_{\gamma^{\prime}}$, the lattice parameter of $\gamma^{\prime}$, as a function of either $y_{i, I}^{\gamma^{\prime}}$ (site fraction of element $i$ in Ni sublattice) or $y_{i, I I}^{\gamma^{\prime}}$ (site fraction of element $i$ in $\mathrm{Al}$ sublattice) depending on whether element $i$ substitutes $\mathrm{Ni}$ or Al. The definitions of the Vegard's coefficients are similar to what has been used earlier [26] and are given by:

$\Gamma_{i}^{\gamma}=\frac{d a_{\gamma}}{d x_{i}^{\gamma}} ; \quad \Gamma_{i, I}^{\gamma^{\prime}}=\frac{d a_{\gamma^{\prime}}}{d y_{i, I}^{\gamma^{\prime}}} ; \quad \Gamma_{i, I I}^{\gamma^{\prime}}=\frac{d a_{\gamma^{\prime}}}{d y_{i, I I}^{\gamma^{\prime}}}$.

While Vegard's coefficients were calculated for $\sim 15$ common alloying elements in Ni-base superalloys, here we present results for only the relevant subset of elements. The coefficients are tabulated in Table 1.

As mentioned earlier, 25 alloy compositions with varying $\mathrm{Re}$ and $\mathrm{Ru}$ contents and with $\mathrm{Ni}-10 \mathrm{Al}$ as the base were evaluated using Thermocalc ${ }^{\mathrm{TM}}$. From this analysis, the composition, $x_{i}^{\gamma}$ of elements in $\gamma$ and site fraction, $y_{i, I}^{\gamma^{\prime}}$ and $y_{i, I I}^{\gamma^{\prime}}$ of elements in $\gamma^{\prime}$ were determined for the 25 compositions at $1050{ }^{\circ} \mathrm{C}$. Using composition and site fraction determined from Thermocalc ${ }^{\mathrm{TM}}$ and Vegard's coefficients determined from first principles calculations, the lattice parameters of the two phases and misfit, $\delta$ between them were evaluated for the 25 compositions. The following formulae used for calculating lattice parameters, assume that the lattice parameter in a multicomponent alloy is a linear combination of the effects of the individual 
Table 1. Vegards coefficients $(\Gamma)$ of alloying elements in $\gamma$ and $\gamma^{\prime}$ (in $\mathrm{Ni}(\mathrm{I})$ and $\mathrm{Al}(\mathrm{II})$ sublattices).

\begin{tabular}{|c|c|c|c|}
\hline Alloying element(i) & $\gamma$ & \multicolumn{2}{|c|}{$\gamma^{\prime}$} \\
\hline & $\Gamma_{i}^{\gamma}$ & $\Gamma_{i, I}^{\gamma^{\prime}}$ & $\Gamma_{i, I I}^{\gamma^{\prime}}$ \\
\hline $\mathrm{Ni}$ & 0 & 0 & -0.045 \\
\hline $\mathrm{Al}$ & 0.188 & 0.266 & 0 \\
\hline $\mathrm{Re}$ & 0.369 & 0.456 & 0.032 \\
\hline $\mathrm{Ru}$ & 0.408 & 0.317 & 0.039 \\
\hline $\mathrm{Hf}$ & 0.769 & 0.948 & 0.169 \\
\hline
\end{tabular}

(a)

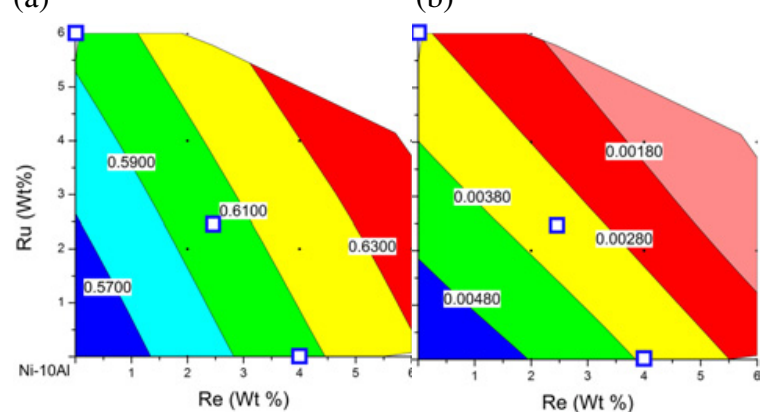

Figure 1. Effect of Re and Ru on (a) vol. fraction of $\gamma^{\prime}$ (b) misfit $(\delta)$ in Ni-10Al-xRe-yRu alloys. The hollow blue squares indicate the Re and Ru levels in the designed model alloys. The alloy also contains $\mathrm{Al}(10 \mathrm{wt} . \%)$ and $\mathrm{Ni}$ (balance). The contour line labels in figures (a) and (b) indicate the volume fraction and misfit along the corresponding contour line.

elements and that there are no interaction terms:

$$
\begin{gathered}
a_{\gamma}=a_{N i}+\sum_{i} \Gamma_{i}^{\gamma} x_{i}^{\gamma} \\
a_{\gamma^{\prime}}=a_{N i_{3} A l}+\sum_{i} \Gamma_{i, I}^{\gamma^{\prime}} y_{i, I}^{\gamma^{\prime}}+\sum_{i} \Gamma_{i, I I}^{\gamma^{\prime}} y_{i, I I}^{\gamma^{\prime}} \\
\delta=2\left(\frac{a_{\gamma^{\prime}}-a_{\gamma}}{a_{\gamma^{\prime}}+a_{\gamma}}\right) .
\end{gathered}
$$

The variation of misfit with $\mathrm{Re}$ and $\mathrm{Ru}$ levels was mapped out at $1050{ }^{\circ} \mathrm{C}$ and this is indicated in the contour map shown in Fig. 1b.

From Fig. 1a, it is clear that in these model alloys, both $\mathrm{Re}$ and $\mathrm{Ru}$ additions result in an increase in the $\gamma^{\prime}$ volume fraction with Re having a greater effect. Figure $1 \mathrm{~b}$ shows that for this range of composition, lattice misfit is positive and addition of both $\mathrm{Re}$ and $\mathrm{Ru}$ are equally effective in decreasing lattice misfit. From these composition domain maps, three alloys were selected with varying amounts of $\mathrm{Re}$ and $\mathrm{Ru}$ and which had a $\gamma^{\prime}$ volume fraction between 0.59 and 0.61 , and misfit between 0.0028 and 0.0038 . All alloys were also constrained to have $\mathrm{Re}$ and $\mathrm{Ru}$ levels less than 6 wt.\% each. In addition, typical trace amounts of $\mathrm{Hf}$ and $\mathrm{C}$ were also added to ensure that the compositions could be processed experimentally to get directionally solidified rods without cracking. Table 2 shows the compositions of model alloys.

To test the validity of the alloy design methodology, the designed alloy compositions were cast, homogenized and heat-treated at $1050^{\circ} \mathrm{C}$. Subsequently microstructural parameters such as $\gamma^{\prime}$ volume fraction and misfit were
Table 2. Composition (in wt.\%) of designed model alloys.

\begin{tabular}{|c|c|c|c|c|c|c|}
\hline Alloy & Al & C & Hf & Ni & Re & Ru \\
\hline Model Re & 10 & 0.07 & 1.4 & bal & 4 & 0 \\
\hline Model ReRu & 10 & 0.07 & 1.4 & bal & 2.5 & 2.5 \\
\hline Model Ru & 10 & 0.07 & 1.4 & bal & 0 & 6 \\
\hline
\end{tabular}

experimentally measured and compared with the designed values. These details are presented next.

\section{Experimental details}

The designed alloys were made into $5 \mathrm{~kg}$ ingots in a vacuum induction melting (VIM) furnace. Rods of dimensions, $\phi=12 \mathrm{~mm}$ and length $=300 \mathrm{~mm}$ were directionally solidified (DS) out of these ingots in a VIM furnace at a withdrawal rate of $24 \mathrm{~cm} / \mathrm{h}$ under a nearly constant thermal gradient.

The as-cast rods exhibit segregation with low melting eutectic pockets rich in $\gamma^{\prime}$ stabilizers and high melting dendrite cores rich in $\gamma$ stabilizers. To have uniform composition throughout the specimen, these rods were subjected to the heat treatments in a Lindberg box-type furnace. The homogenization schedule is as follows: $1298^{\circ} \mathrm{C} / 2 \mathrm{hrs}(\operatorname{argon}) \rightarrow 1303^{\circ} \mathrm{C} / 2 \mathrm{hrs}(\operatorname{argon}) \rightarrow$ $1308^{\circ} \mathrm{C} / 20 \mathrm{hrs}$ (argon) $\rightarrow 1310^{\circ} \mathrm{C} / 55 \mathrm{hrs}$ (vacuum). After homogenization, the specimens were water-quenched. Since the microstructural parameters were designed with precipitate fraction and misfit corresponding to that at $1050^{\circ} \mathrm{C}$, samples were aged at $1050^{\circ} \mathrm{C}$ for $4 \mathrm{hrs}$ and then water-quenched. The volume fraction and composition of the phases at $1050{ }^{\circ} \mathrm{C}$ was expected to be retained at room temperature.

Specimens were prepared for Scanning Electron Microscopy (SEM) and Electron Probe MicroAnalysis (EPMA) by grinding using a series of ever-finer emery papers. Subsequently, they were polished to a surface roughness of $0.25 \mu \mathrm{m}$ using a diamond suspension. Final polishing was done with $0.04 \mu \mathrm{m}$ colloidal silica suspension in BEUHLER Vibromet $2^{\mathrm{TM}}$ polisher. FEI Quanta $^{\mathrm{TM}}$ ESEM was used to image the samples in the Back Scattered Electrons (BSE) mode. EPMA was done on a JEOL JXA-8530F using wavelength dispersive spectroscopy for quantifying composition and for imaging. Precipitate area fractions were evaluated from SEM-BSE images by standard metallography procedures using the ImageJ software [27].

Lattice parameter measurements were done by analysing the (001), (002) and (003) peaks determined from a polished section of the aged rods. The patterns were obtained from RIGAKU Smartlab Xray diffractometer. $\mathrm{Cu} K \alpha_{1}$ radiation was used after stripping the $\mathrm{K} \alpha_{2}$ contributions using a $\mathrm{Ge}(220) \times 2$ bounce monochromator. Slow scans were done with a step size as small as $0.0012^{\circ}$ and a scan rate of $0.1^{\circ} \mathrm{min}$. The $(001)$ and $(003)$ peaks of $\gamma^{\prime}$ were used to obtain the lattice parameter of $\gamma^{\prime}$. Using this measure of the $\gamma^{\prime}$ lattice parameter, the (002) peak was deconvoluted to separate out contributions from $\gamma$ and $\gamma^{\prime}$. Gaussian or Lorenzian peak profiles were assumed. From this, the lattice parameter of $\gamma$ was determined. Using these lattice parameters, the constrained misfit 


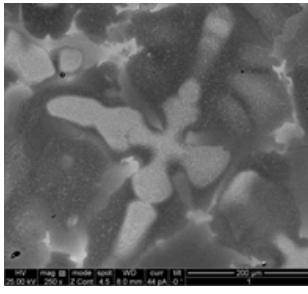

(a)

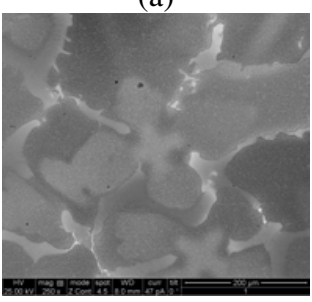

(c)

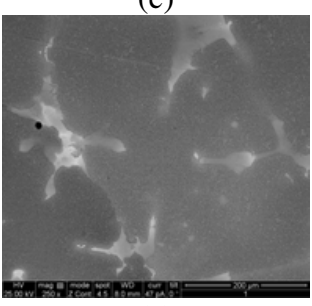

(e)

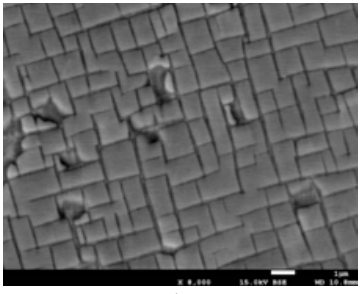

(b)

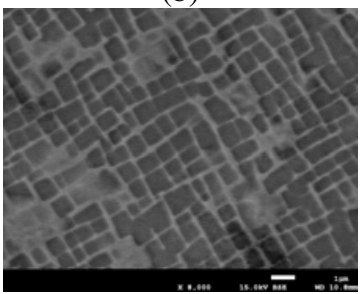

(d)

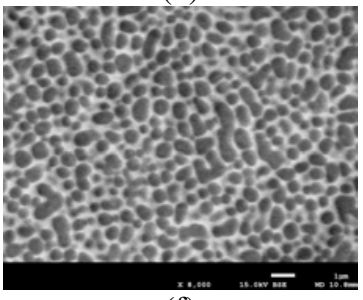

(f)
Figure 2. Microstructures of as-cast (left) and aged (right) samples in (a,b) Model Re, (c,d) Model ReRu, (e,f) Model Ru. Note that the aged samples are imaged at higher magnification.

$(\varepsilon)$ was determined. The constrained misfit is the misfit obtained when lattice parameters are measured with $\gamma^{\prime}$ embedded in $\gamma$. The constrained misfit is distinct from the unconstrained misfit $(\delta)$ which was defined earlier (Eq. (4)). Previous studies have suggested that these misfits are related to one another by $\kappa=(\varepsilon / \delta)$, a material and microstructure dependent parameter. Assuming isotropic elasticity, Mott and Nabarro [28] have shown that for a spherical precipitate of bulk modulus $K_{p}$ embedded in an infinite matrix of shear modulus, $G_{m}, \kappa=1 /(1+$ $\left.4 G_{m} / 3 K_{p}\right)$. Since it is known that the moduli of the two phases are comparable [29], assuming identical moduli for $\gamma$ and $\gamma^{\prime}$ and a Poisson's ratio of 0.33 , results in $\kappa=0.66$. Grose and Ansell [30] have confirmed that for $\gamma^{\prime}$ spherical precipitates, $\kappa=0.64$ indicating that the isotropic assumption is valid. However, Glatzel and FellerKniepmeier [31] have shown that precipitate morphology also affect the value of the constrained misfit and so for cuboidal precipitates $\left(\gamma^{\prime}\right.$ vol. frac. $\left.=60 \%\right), \kappa=0.85$. In the present study, the unconstrained misfit $(\delta)$ was obtained from the constrained misfit $(\varepsilon)$ derived from $\mathrm{X}$ ray studies using $\kappa$ values of both 0.64 and 0.85 , as they represent different bounds.

\section{Results}

Figure 2 shows the microstructures of the as-cast and aged $\left(1050^{\circ} \mathrm{C}, 4 \mathrm{hrs}\right)$ specimens. The as-cast microstructures exhibit segregation. After homogenization the alloy composition is uniform within the dendrite arms as confirmed by EPMA. In the aged microstructures of Model ReRu and Model Ru, the dark phase (containing

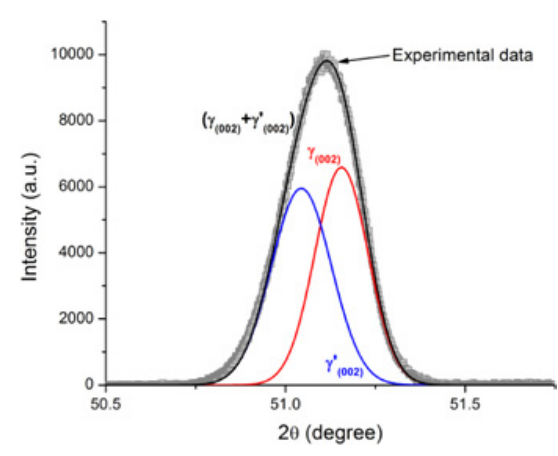

Figure 3. The (002) peak and its deconvolution into the individual $\gamma_{(002)}^{\prime}$ and $\gamma_{(002)}$ components for Model Re. The same procedure was followed for the other alloys.

higher $\mathrm{Al}$ ) is $\gamma^{\prime}$, while the brighter background is the $\gamma$ matrix. Attention is also drawn to the difference in contrast in Model Re where the background is darker than the precipitates. Using several such images, the volume fraction of $\gamma^{\prime}$ was determined for all three alloys. The values are tabulated in Table 3. Model Re has highest volume fraction (and precipitate size), while Model $\mathrm{Ru}$ has the lowest volume fraction (and precipitate size). Additionally on going from Model Re to Model Ru, the precipitate morphology changes from cuboidal to spherical. These results are discussed in the next section.

Figure 3 shows an example of the (002) peak and its deconvolution into the individual $(002)_{\gamma^{\prime}}$ and $(002)_{\gamma}$ components. In all cases, it is observed that the $(002)_{\gamma}$ peak occurs at higher $2 \theta$ values than $(002)_{\gamma^{\prime}}$ suggesting that the lattice parameter of $\gamma^{\prime}$ is greater than that of $\gamma$ indicating that these alloys have a positive misfit, as expected from alloy design. The measured values of the unconstrained misfit are compared against the predicted value of unconstrained misfit in Table 3.

\section{Discussion}

While the match between the measured $\gamma^{\prime}$ volume fractions and the designed values is excellent for the Model $\mathrm{Ru}$ alloy, the measured $\gamma^{\prime}$ volume fractions in Model Re and Model ReRu are higher than the designed values. A possible source of mismatch in volume fraction could be compositional inhomogeneities resulting from inadequate homogenization; it was observed that some eutectic pools remained even after homogenization. Local EPMA measurements confirmed that the non-eutectic regions (i.e. the homogenized dendrite arms and cores), where $\gamma^{\prime}$ volume fraction was measured, were leaner in $\mathrm{Al}$ (by $\sim 1 \mathrm{wt} . \%$ ) and richer in $\mathrm{Ni}$ (by $\sim 2 \%$ ) than the designed alloy compositions in Model Re and Model ReRu. The $\mathrm{Re} / \mathrm{Ru}$ levels in the dendrite arms matched the designed levels closely. $\gamma^{\prime}$ volume fraction and misfit was predicted for dendrite arm composition using the same procedure as used for the designed alloy composition and this is also indicated in Table 3. The match between the predicted and achieved $\gamma^{\prime}$ volume fraction and misfit is even poorer when dendrite arm composition is used. Clearly, the mismatch between design and measured values cannot be reconciled simply by accounting for compositional inhomogeneities. 
Table 3. Validation of predicted microstructural parameters against experimental data. The predicted values in parenthesis and bold are derived from the homogenized dendrite arm/core compositions. Measured $\delta$ values were obtained from $\varepsilon$ using different values of $\kappa$.

\begin{tabular}{|c|c|c|c|}
\hline \multirow{2}{*}{ Alloy } & \multirow{2}{*}{$\gamma^{\prime}$ morphology } & \multicolumn{2}{|c|}{$\gamma^{\prime}$ Volume fraction } \\
\hline & & Predicted & Achieved \\
\hline Model Re & Cuboidal & $\begin{array}{c}0.605 \\
(\mathbf{0 . 4 4 2})\end{array}$ & $0.783( \pm 0.029)$ \\
\hline Model ReRu & Cuboidal & $\begin{array}{c}0.602 \\
(\mathbf{0 . 4 4 6 )}\end{array}$ & $0.670( \pm 0.043)$ \\
\hline Model Ru & Spherical & \begin{tabular}{c|}
0.596 \\
$(\mathbf{0 . 5 9 1 )}$ \\
\end{tabular} & $0.626( \pm 0.053)$ \\
\hline \multirow{3}{*}{ Alloy } & \multicolumn{3}{|c|}{ Unconstrained misfit $(\delta)$} \\
\hline & \multirow{2}{*}{ Predicted } & \multicolumn{2}{|c|}{ Achieved } \\
\hline & & $\kappa=0.64$ & $\kappa=0.85$ \\
\hline Model Re & $\begin{array}{c}0.0037 \\
(\mathbf{0 . 0 0 4 0 )}\end{array}$ & $\begin{array}{c}0.0021 \\
\left( \pm 2 \times 10^{-4}\right)\end{array}$ & $\begin{array}{c}0.0016 \\
\left( \pm 1 \times 10^{-4}\right)\end{array}$ \\
\hline Model ReRu & $\begin{array}{c}0.0033 \\
(\mathbf{0 . 0 0 3 5})\end{array}$ & $\begin{array}{c}0.0011 \\
\left( \pm 1 \times 10^{-4}\right)\end{array}$ & $\begin{array}{c}0.0009 \\
\left( \pm 1 \times 10^{-4}\right)\end{array}$ \\
\hline Model Ru & $\begin{array}{c}0.0029 \\
(\mathbf{0 . 0 0 3 5})\end{array}$ & $\begin{array}{c}0.0010 \\
\left( \pm 1 \times 10^{-4}\right)\end{array}$ & $\begin{array}{c}0.0007 \\
\left( \pm 1 \times 10^{-4}\right)\end{array}$ \\
\hline
\end{tabular}

In light of this, it is suggested that ThermoCalc predictions of composition and volume fraction (from either the alloy or the dendrite composition) are in error due to inadequacies in the thermodynamic database. Experimental data on partition coefficient and $\gamma^{\prime}$ volume fraction in selected ternary/quarternary and multicomponent systems are compared against ThermoCalc predictions in Table 4. The partition coefficient, $k_{x}^{\gamma^{\prime} / \gamma}$ is defined as the ratio of composition of species $\mathrm{X}$ in $\gamma^{\prime}$ to $\gamma$. It is noted that while some ThermoCalc predictions of volume fraction and partition coefficient match the experimental data, the match is poor for others. This is true not only for $\mathrm{Re}$ and $\mathrm{Ru}$, but also for other alloying elements (e.g., W and $\mathrm{Cr}$ ). The partitioning coefficient for $\mathrm{Re}$, in particular, is not in agreement with experimental observations with the mismatch being pronounced in ternary/quarternary systems. ThermoCalc predicts that Re should strongly partition to $\gamma$. Instead, it has been experimentally observed that Re in small amounts in ternary/quarternary systems [3] partitions instead to $\gamma^{\prime}$. Additionally, while $\operatorname{Re}$ does partition to $\gamma$ in multicomponent systems, it doesn't do so as strongly as predicted. The divergent behaviour of Re in ternary/quarternary and multicomponents systems is not captured adequately by the ThermoCalc database.

It is likely that in Model Re and Model ReRu alloys in this study, Re partitions to $\gamma^{\prime}$ and stabilises the phase resulting in a significantly higher volume fraction. While partition coefficients were not measured in the present study, BSE contrast in Fig. 2b provides vital clues. The appearance of $\gamma^{\prime}$ as the brighter phase, despite its higher Al content suggests strong Re partitioning to $\gamma^{\prime}$. While thermodynamic predictions of volume fractions (Table 3) do not match the measured values in Re-containing alloys, the match is excellent in Model Ru suggesting that the $\mathrm{Ru}$ description in the database is robust. This is consistent with the observation that the predicted and measured partition coefficients of Ru are in good agreement (Table 4).
Table 4. Experimental and ThermoCalc predicted data (parenthesis, bold) of partitioning coefficient of elements and volume fractions of $\gamma^{\prime}$ in selected alloy systems.

\begin{tabular}{|l|c|c|c|}
\hline \multirow{2}{*}{ Alloy (wt\%) } & \multicolumn{2}{|c|}{ Partition coefficient } & Vol. frac. of $\gamma^{\prime}$ \\
\cline { 2 - 4 } & $k_{W}^{\gamma^{\prime} / \gamma}$ & $k_{C r}^{\gamma^{\prime} / \gamma}$ & \\
\hline Ni-8Al-3W [3] & $1.46(\mathbf{3 . 8 9})$ & - & - \\
\hline Ni-8.2Al-2.9Cr- & $1.00(\mathbf{1 . 4 9 )}$ & $0.32(\mathbf{0 . 4 2})$ & $0.95(\mathbf{0 . 7 9 )}$ \\
7.6W [33] & & & \\
\hline Ni-7.1Al-3.8Cr- & $0.82(\mathbf{0 . 7 3})$ & $0.02(\mathbf{0 . 3 0})$ & $0.30(\mathbf{0 . 9 2})$ \\
22.3W [33] & & & \\
\hline & $k_{R e}^{\gamma^{\prime} / \gamma}$ & $k_{R u}^{\gamma^{\prime} / \gamma}$ & \\
\hline Ni-8Al-3Re [3] & $1.85(\mathbf{0 . 2 1})$ & - & - \\
\hline Ni-8Al-3Ru [3] & - & $0.46(\mathbf{0 . 4 8})$ & - \\
\hline Ni-8Al-3Re-3Ru [3] & $1.03(\mathbf{0 . 2 1})$ & $0.61(\mathbf{0 . 4 7})$ & - \\
\hline Ni-4.9Al-3.9Ti- & & & \\
8.2Cr-4.1Co- & $0.29(\mathbf{0 . 0 4})$ & $0.49(\mathbf{0 . 3 1})$ & $0.54 \mathbf{( 0 . 6 5 )}$ \\
2.5Mo-1.6Ta- & & & \\
3Re-3Ru [12] & & & \multirow{2}{*}{$0.51 \mathbf{( 0 . 6 4 )}$} \\
\hline Ni-4.9Al-3.9Ti- & & & \\
8.2Cr-4.1Co- & $0.11(\mathbf{0 . 0 4})$ & - & \\
2.5Mo-1.6Ta- & & & \\
3Re [12] & & &
\end{tabular}

Discrepancies in ThermoCalc predicted volume fractions of $\gamma^{\prime}$ and TCP phase have also been reported by Pyczak et al. [12] and Heckl et al. [32] in Rebearing alloys. Similar issues in the predictions of phases, phase fractions and phase compositions have been previously identified in Ni-Al-Cr-W systems [3335] and this has resulted in the refinement of the relevant database, and hence improved thermodynamic descriptions in subsequent versions. It is hoped that the present results will inform the refinement of the $\mathrm{Re}$ description in ternary/quarternary systems.

The change in precipitate morphology from cuboidal to spherical on decreasing the $\mathrm{Re} / \mathrm{Ru}$ ratio is similar to observations by Heckl et al. [4]. This is related to the effect of $\mathrm{Re} / \mathrm{Ru}$ on misfit. Model Re has a high misfit which is consistent with cuboidal precipitates, while Model $\mathrm{Ru}$ has a low misfit close to zero and this is consistent with spherical precipitates. Thus the measured misfit is consistent with the observed precipitate morphology suggesting that the methodology for measuring misfit is reasonably accurate. However, experimentally measured misfit values are lower than the predicted values from dendrite arm compositions for all alloys. In Model Re and Model $\mathrm{ReRu}$, it is likely due to the use of incorrect phase composition (derived from ThermoCalc) for predicting lattice parameters and misfit. The lack of agreement in misfit in the case of Model Ru is puzzling since in this case ThermoCalc predictions of phase fractions are good and thus one expects phase compositions to be accurate as well. This suggests that perhaps Vegard's coefficients for $\mathrm{Ru}$ are in error.

Since it appears that thermodynamic descriptions are more accurate in multicomponent systems (Table 4), it may be possible to design multicomponent alloys with designed microstructural parameters. However, in multicomponent alloys, application of Vegard's coefficients (derived from binary and pseudo-binary systems), is questionable given 
that there can be significant interactions between alloying elements. It would be interesting to conduct a study similar to the present one to probe the appropriateness of thermodynamic databases and the Vegard's coefficients in multicomponent systems.

\section{Summary}

Using a computational approach involving thermodynamic and first principles calculations, model alloys were designed with varying levels of $\mathrm{Re}$ and $\mathrm{Ru}$, but which were constrained to have the same phase fractions and lattice misfit across the alloys. Experimental results suggest that there are deviations from the designed values in both volume fraction and misfit in Re containing alloys due to inadequacies in the thermodynamic databases used. This work suggests that computational design of alloy systems from thermodynamic and first principles calculations is still at its infancy even in ternary and quaternary alloy systems.

The authors would like to acknowledge the financial support provided by Defence Research and Development Organization (DRDO), Government of India, and material and method support from scientists at DMRL, Hyderabad. KVV acknowledges the $\mathrm{PhD}$ fellowship provided by University Grants commission (UGC), Government of India.

\section{References}

[1] C.T. Liu, D.P. Pope, in Intermetallic. Compounds. Structural Applications of Intermetallic Compounds 55 (John Wiley \& Sons, Ltd, 2002)

[2] D.R. Lide, CRC Handbook of Chemistry and Physics 87 (CRC Press, 2007)

[3] A. Volek, F. Pyczak, R.F. Singer, H. Mughrabi, Scr. Mater. 52, 141 (2005)

[4] A. Heckl, R. Rettig, R.F. Singer, Adv. Mater. Res. 278, 339 (2011)

[5] A. Heckl, S. Neumeier, M. Goken, R.F. Singer, Mater. Sci. Eng. A 528, 3435 (2011)

[6] R.A. Hobbs, L. Zhang, C.M.F. Rae, S. Tin, Mater. Sci. Eng. A 489, 65 (2008)

[7] F. Pyczak, B. Devrient, F.C. Neuner, H. Mughrabi, Acta Mater. 53, 3879 (2005)

[8] A.K. Sinha, Topologically Close-Packed Structures of Transition Metal Alloys (Pergamon Press, Oxford, New York, 1972)

[9] C.M.F. Rae, R.C. Reed, Acta Mater. 49, 4113 (2001)

[10] A.C. Yeh, C.M.F. Rae, S. Tin, Proceedings of Tenth International Symposium on Superalloys, 677 (The Minerals, Metals and Materials Society (TMS), Warrendale, PA, 2004)
[11] A.P. Ofori, C.J. Humphreys, S. Tin and C.N. Jones, in Proceedings of Tenth International Symposium on Superalloys, 1319 (The Minerals, Metals and Materials Society (TMS), Warrendale, PA, 2004)

[12] F. Pyczak, S. Neumeier, M. Göken, Mater. Sci. Eng. A 527, 7939 (2010)

[13] M.S.A. Karunaratne, P. Carter, R.C. Reed, Mater. Sci. Eng. A 281, 229 (2000)

[14] B. Sundman, B. Jansson, J.O. Andersson, Calphad 9, 153 (1985)

[15] G. Kresse, J. Hafner, Phys. Rev. B 47, 558 (1993)

[16] G. Kresse, J. Furthmüller, Phys. Rev. B 54, 11169 (1996)

[17] G. Kresse, J. Hafner, Phys. Rev. B 49, 14251 (1994)

[18] G. Kresse, J. Furthmüller, Comput. Mater. Sci. 6, 15 (1996)

[19] T. Murakumo, T. Kobayashi, Y. Koizumi, H. Harada, Acta Mater. 52, 3737 (2004)

[20] Y. Koizumi, T. Kobayashi, T. Yokokawa, Jianxin Zhang, M. Osawa, H. Harada, Y. Aoki and M. Arai, in Proceedings of Tenth International Symposium on Superalloys 35 (The Minerals, Metals and Materials Society (TMS), Warrendale, PA, 2004)

[21] MedeA version 2.6.6, Materials Design Inc, Angel Fire, NM, USA (2009)

[22] M. Methfessel, A.T. Paxton, Phys. Rev. B 40, 3616 (1989)

[23] J.P. Perdew, K. Burke, M. Ernzerhof, Phys. Rev. Lett. 77, 3865 (1996)

[24] J.P. Perdew, K. Burke, M. Ernzerhof, Phys. Rev. Lett. 78, 1396 (1997)

[25] K.V. Vamsi, S. Karthikeyan, Proceedings of Twelfth International Symposium on Superalloys, 521 (The Minerals, Metals and Materials Society (TMS), Warrendale, PA, 2012).

[26] T. Wang, L.-Q. Chen, Z.-K. Liu, Mater. Sci. Eng. A 431, 196 (2006)

[27] Image J, "http://imagej.nih.gov/ij/"

[28] N.F. Mott, F.R.N. Nabarro, Proc. Phys. Soc. 52, 86 (1940)

[29] T.M. Pollock, A.S. Argon, Acta Metall. Mater. 42, 1859 (1994)

[30] D.A. Grose, G.S. Ansell, Metall. Trans. A 12, 1631 (1981)

[31] U. Glatzel, M. Feller-Kniepmeier, Scr. Metall. 23, 1839 (1989)

[32] A. Heckl, S. Neumeier, S. Cenanovic, M. Göken, R.F. Singer, Acta Mater. 59, 6563 (2011)

[33] J. Buršık, P. Brož, R. Picha, Intermetallics 11, 483 (2003)

[34] P. Brož, J. Buršík, Z. Stará, Monatshefte Für Chem. Chem. Mon. 136, 1915 (2005)

[35] J. Popovič, P. Brož, J. Buršík, Intermetallics 16, 884 (2008) 